\title{
Interleukin-10 overexpression improves the function of endothelial progenitor cells stimulated with TNF- $\alpha$ through the activation of the STAT3 signaling pathway
}

\author{
YING WANG, QINGZHONG CHEN, ZHUHONG ZHANG, FENG JIANG, XIANGDA MENG and HUA YAN \\ Department of Ophthalmology, Tianjin Medical University General Hospital, Tianjin 300052, P.R. China
}

Received August 2, 2014; Accepted December 4, 2014

DOI: $10.3892 /$ ijmm.2014.2034

\begin{abstract}
Lentivirus vector-interleukin-10 green fluorescent protein (LV-IL-10-GFP) was transfected into endothelial progenitor cells (EPCs) in the present study. The aim was to detect the function of IL-10-modified EPCs and analyze the molecular mechanism. EPCs were cultured and identified by fluorescent labeling with the von Willebrand factor antibody, vascular endothelial growth factor (VEGF) receptor, Ulex europaeus agglutinin-1 and acetylated low-density lipoprotein. Subsequently, EPCs were transfected with LV-IL-10-GFP and lentivirus vector-noncontain-GFP as the control group. Enzyme-linked immunosorbent assay (ELISA) was used to detect the concentrations of cytokines in the supernatant with or without tumor necrosis factor- $\alpha$ (TNF- $\alpha)$. All types of cells were assessed by a tube formation assay, adhesion assay and migration assay induced with or without TNF- $\alpha$. Cell cycle was assessed by flow cytometry. Western blot analysis was applied to detect the expression of proteins in the cells. ELISA analysis showed that the levels of TNF- $\alpha$ and IL- 8 in the supernatant without TNF- $\alpha$ significantly decreased in EPC-LV-IL-10-GFP $(\mathrm{P}<0.05$ for all). By contrast, the levels of IL-10 and VEGF were contrasting in association with these. The concentrations of cytokines in the supernatant with TNF- $\alpha$ were consistent to the supernatant without TNF- $\alpha$. There was no statistically significant difference in the average number of EPCs undergoing migration, adhesion, total length and cell growth among the EPC, EPC-LV-IL-10-GFP and EPC-LV-NC-GFP groups without TNF- $\alpha$. Further study showed that EPC-LV-IL-10-GFP with TNF- $\alpha$ significantly enhanced EPC migration, adhesion and promoted tube formation $(\mathrm{P}<0.05$ for all). Western blot analysis revealed that the expression of VEGF, matrix metallopeptidase-9 and phosphorylated-signal transducer and activator of transcription 3 (p-STAT3) significantly increased
\end{abstract}

Correspondence to: Dr Hua Yan, Department of Ophthalmology, Tianjin Medical University General Hospital, 154 Anshan Road, Tianjin 300052, P.R. China

E-mail: phuayan2000@163.com

Key words: interleukin-10, endothelial progenitor cells, signal transducer and activator of transcription 3 signaling pathway in the EPC-LV-IL-10-GFP group. Conversely, STAT-3 expression decreased in the EPC-LV-IL-10-GFP group. The present study suggested that overexpression of IL-10 had no effect on migration, adhesion, tubule formation and cell growth of EPCs without TNF- $\alpha$. Furthermore, in EPCs stimulated with TNF- $\alpha$, the overexpression of IL-10 improved EPC function, including migration, adhesion and tubule formation by activating the STAT3 signal pathway.

\section{Introduction}

Bone marrow (BM)-derived endothelial progenitor cells (EPCs) play a crucial role in neovascularazation. In the past decades, the regenerative potential of EPCs in the injured endothelia, including myocardial ischemia (1), carotid artery injury (2) and limb ischemia (3), has been extensively investigated. However, it is now known that the hostile wound environment in settings, such as chronic wound healing and ischemic myocardial infarction characterized by hypoxia, and increased inflammation and increased free radicals, has an adverse effect on the survival and function of transplanted EPCs, thereby compromising their full therapeutic benefit (4). In addition, there is a study that heart failure patients with high circulating levels of tumor necrosis factor- $\alpha$ (TNF- $\alpha$ ), a potent pro-inflammatory cytokine, are associated with significantly lower EPC counts as compared to patients treated with a TNF- $\alpha$ inhibitor (5). Therefore, modulation of the local tissue microenvironment by anti-inflammatory factors can confer an improved stem cell survival and function, and improved clinical efficacy.

Interleukin-10 (IL-10), a potent anti-inflammatory cytokine, attenuates inflammatory response and suppresses various pro-inflammatory mediators (6-8). IL-10 plays a role not only in immunoregulation and inhibition of pro-inflammatory cytokine synthesis, but also in directly regulating the growth and survival of noninflammatory cells. Several studies have demonstrated that BM-mononuclear cells (MNCs), as well as mesenchymal stem cells (MSCs), have the ability to immunoregulate and improve tissue repair through IL-10 secretion (9-11). A further study by Krishnamurthy et al (12) demonstrated that IL-10 has a role on EPC mobilization following myocardial injury. Therefore, we hypothesized that IL-10 modulates EPC biology and enhances its function via activation of the signal transducer and activator of transcription 3 (STAT3) signaling pathway. 


\section{Materials and methods}

Major reagents. Lentivirus vector-IL-10-green fluorescent protein (LV-IL-10-GFP) and LV-noncontain-GFP (LV-NC-GFP) were obtained from Xi'An Kewei Biological Technology Company (Xi'an, China). Horseradish peroxidase affinipure goat anti-rabbit immunoglobulin G (E030120-02) and goat anti-mouse (E030110-02) were purchased from EarthOx, LLC (San Francisco, CA, USA). The anti-matrix metallopeptidase-9 (MMP-9) antibody (ab58803), anti-vascular endothelial growth factor (VEGF) antibody (ab46154), anti-STAT3 antibody (ab11935) and anti-fluorescein isothiocyanate (FITC)-conjugated von Willebrand factor (vWF) antibody (ab8822) were purchased from Abcam (Cambridge, UK). Anti-phycoerythrin (PE)-conjugated VEGF receptor (VEGFR2) antibody (YM-0565R) was purchased from Shanghai Yanmeng Biological Technology Co. (Shanghai, China). Phosphorylated-STAT3 (p-STAT3; \#9145s) was purchased from Cell Signaling Technology (Danvers, MA, USA). Electrochemiluminescence (ECL) was obtained from Millipore Corporation (Billerica, MA, USA).

Animals. Postnatal 7-9-day-old male Wistar rats were purchased from the Academy of Military Medical Sciences (Beijing, China). All the animals in the study were cared, used and treated in strict accordance to the ARVO statement for the use of animals in ophthalmic and vision research.

Isolation and culture of EPCs. BM-derived EPCs were cultured according to established methods $(13,14)$. In brief, postnatal 7-9-day-old rats were sacrificed by cervical dislocation and the tibias were extracted. The BM was flushed out of the tibias with sterile phosphate-buffered saline (PBS) using a syringe, and MNCs were isolated by Ficoll gradient centrifugation. Following washing twice with PBS, MNCs were seeded on a human fibronectin (FN; Sigma-Aldrich, St. Louis, MO, USA) coated 6-well plate at a density of $5 \times 10^{6}$ cell/well and cultured in endothelial cell growth medium-2 (EGM-2; Lonza, Walkersville, $\mathrm{MD}, \mathrm{USA}$ ) at $37^{\circ} \mathrm{C}, 5 \% \mathrm{CO}_{2}$. EGM-2 was changed every two days and non-adherent cells were removed. After 7 days in culture, cells were identified by fluorescent-activated cell sorting (FACS; Calibur flow cytometer, Becton Dickinson, CA, USA) and immunofluorescence.

Characterization of BM-derived EPCs. Identification of EPCs was first by fluorescent labeling, direct binding of FITC-conjugated Ulex europaeus agglutinin (UEA-1; Sigma-Aldrich) and uptake of 1,1'-dioctadecyl-3,3,3',3'-tetramethylindocarbocyanine percholate (DiI)-labeled acetylated low-density lipoprotein (acLDL; Biomedical Technologies, Stoughton, MA, USA) were performed. For these assays, the cells were first incubated with DiI-ac-LDL $(10 \mu \mathrm{g} / \mathrm{ml}$ in EBM-2 medium) at $37^{\circ} \mathrm{C}$ for $4 \mathrm{~h}$ and were subsequently fixed with $4 \%$ paraformaldehyde (PFA) and counterstained with UEA-1 (10 $\mu \mathrm{g} / \mathrm{ml}$ in $0.9 \%$ saline). Subsequently, images were captured under an inverted fluorescence microscope (Olympus, Tokyo, Japan).

For extracellular labeling of vWF and VEGFR2, cells were fixed with 4\% PFA for 30 mins and blocked in 5\% bovine serum albumin and labeled sequentially with anti-vWF antibody conjugated to FITC and anti-VEGFR2 antibody conjugated to PE. The cells were subsequently washed three times in PBS, counterstained with diamidino-phenyl-indole for 5 mins. Following washing, images were captured by fluorescence microscopy.

Lentiviral transduction. Following identification, EPCs were transfected with LV-IL-10-GFP (EPC-LV-IL-10-GFP) and LV-NC-GFP (EPC-LV-NC-GFP) as the control group, whereas the LV expressing enhanced GFP was used to measure transduction efficiency. To achieve optimal gene transfer, polybrene (1:1000; Biomedical Technologies) was used, and the medium was changed $4 \mathrm{~h}$ later. Two days later, transfection efficiency was observed under an inverted fluorescence microscope and the transfected cells were used for all the experiments. Before being used, a section of the cells were starved for $12 \mathrm{~h}$ and were subsequently stimulated with recombinant rat TNF- $\alpha$ (PeproTech, Rocky Hill, NJ, USA) at $10 \mathrm{ng} / \mathrm{ml}$, and the medium was changed $12 \mathrm{~h}$ after stimulation.

Assessment of TNF- $\alpha, I L-10, I L-8$ and VEGF by ELISA. The concentrations of TNF- $\alpha$, IL-10, IL- 8 and VEGF in EGM-2 medium were measured by rat enzyme-linked immunosorbent assay (ELISA) kits (R\&D Systems, Inc., Minneapolis, MN,USA) according to the manufacturer's instructions. Subsequently, all the groups of supernatants were collected and the assays for the levels of TNF- $\alpha$, IL-10, IL-8 and VEGF were performed.

Migration assay of IL-10-modified EPCs. Cell migration was tested in Transwell 12-well plates (Corning, New York, NY, USA). Cells were digested and re-suspended in serum-free EGM-2 medium, and subsequently, $2 \times 10^{4}$ cells were loaded into the upper chambers. The lower chamber was filled with EGM-2 medium. After $6 \mathrm{~h}$ of incubation at $37^{\circ} \mathrm{C}$, cells were stained with crystal violet and counted in 3 random fields (magnification, x200) in each well.

Adhesion assay of IL-10-modified EPCs. Cell adhesion was examined in 96-well plates coated overnight $\left(4^{\circ} \mathrm{C}\right)$ with $\mathrm{FN}$. Cells were digested, re-suspended and plated at a concentration of $5 \times 10^{4}$ cells/well in $100 \mathrm{ml}$ of serum-free EGM-2 medium. Adhesion was carried out for $4 \mathrm{~h}$ at $37^{\circ} \mathrm{C}$. Removal of non-adherent cells was achieved by two washing steps with serum-free EGM-2 medium. Adhesion was quantified by counting adherent cells in 3 random fields (magnification, $\mathrm{x} 200)$ in each well.

Tube formation assay of IL-10-modified EPCs. Serum-starved cells $\left(1 \times 10^{5}\right)$ were seeded onto a matrigel (BD Bioscience, San Jose, CA, USA)-coated plate in EGM-2 medium and incubated at $37^{\circ} \mathrm{C}$ for $10 \mathrm{~h}$. To quantify the length of newly-formed tubes, photomicrographs in 3 random fields (magnification, x100) per well were captured, and the total tubule lengths were measured using WimTube Image software (Wimasis GmbH, Munich, Germany).

Cell cycle analysis of IL-10-modified EPCs by flow cytometry. Cells $\left(1 \times 10^{6}\right)$ were digested, re-suspended and added to ice-cold methanol for fixation and stored at $4^{\circ} \mathrm{C}$. Samples were warmed to room temperature and rehydrated by rinsing twice in PBS. 


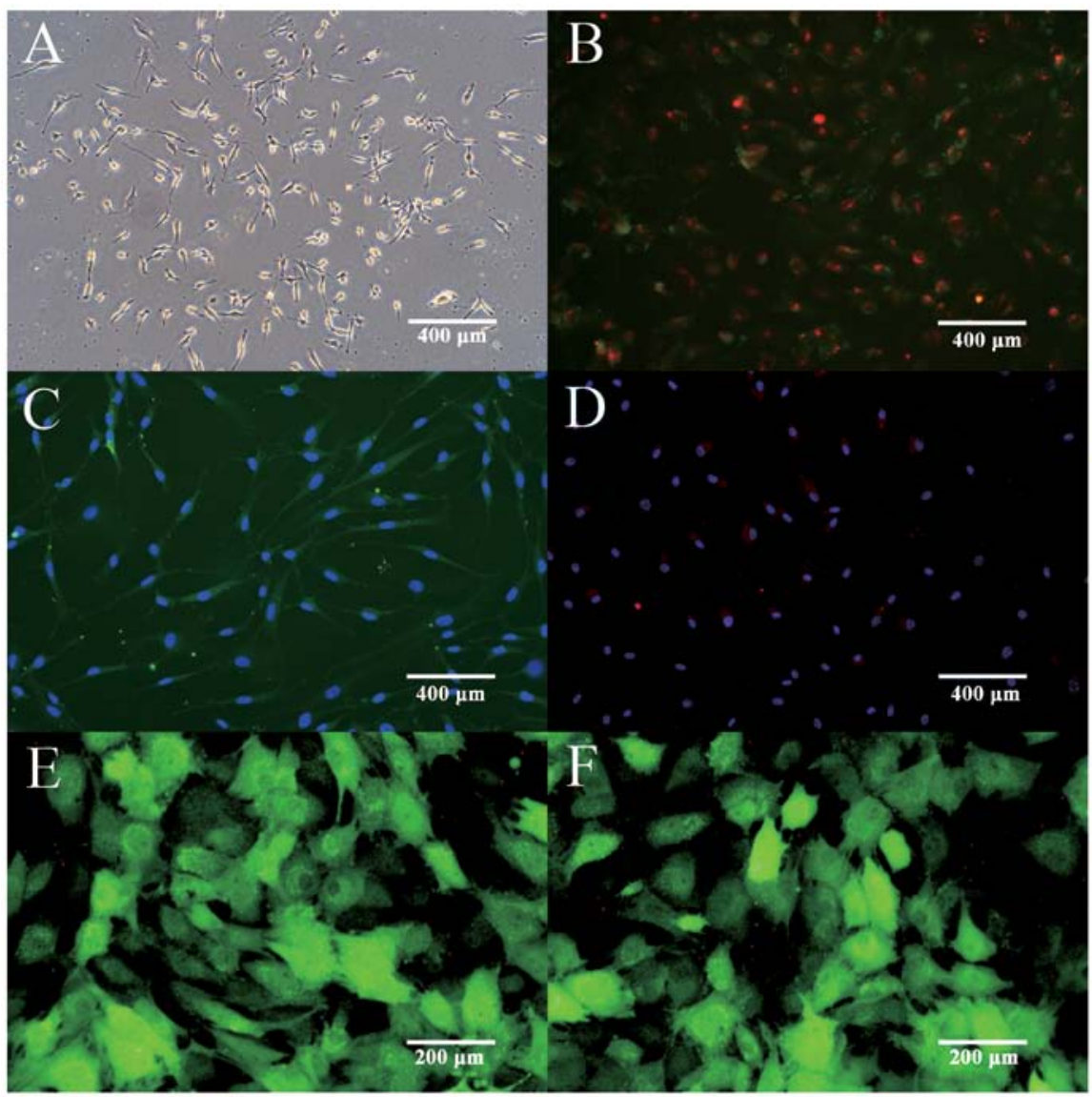

Figure 1. Characterization of EPCs and transfected EPCs. (A) Isolation of EPCs exhibited a spindle appearance after culturing for $72 \mathrm{~h}$ (magnification, x100). Fluorescence microscopy illustrated that adherent cells were positive for uptake of Dil-acLDL and FITC-UEA-1. (B) The double-positive cells were recognized as EPCs (magnification, x100). (C) Immunoflurescent staining demonstrated that adherent cells had positive expression of endothelial marker FITC-vWF (magnification, x100) and (D) PE-VEGFR2 (magnification, x100). Fluorescence microscopy showed EPCs expression of GFP following transfection with (E) LV-IL-10-GFP (magnification, x200) and (F) LV-NC-GFP (magnification, x200). EPC, endothelial progenitor cells; Dil-acLDL, 1,1'-dioctadecyl-3,3,3',3'-tetramethylindocarbocyanine percholate-labeled acetylated low-density lipoprotein; FITC-UEA-1, fluorescein isothiocyanate-Ulex europaeus agglutinin; vWF, von Willebrand factor; PE-VEGFR2, phycoerythrin-vascular endothelial growth factor receptor 2; GFP, green fluorescent protein; LV, lentivirus vector; IL-10, interleukin-10; NC, noncontain.

Samples were centrifuged $\left(250 \mathrm{xg}, 10 \mathrm{~min}\right.$ at $\left.4^{\circ} \mathrm{C}\right)$ and incubated in $500 \mu 1 \mathrm{PI} / \mathrm{RNase}$ staining buffer (BD Biosciences) for $15 \mathrm{~min}$. Then the cells were analyzed on a flow cytometry. The data were analyzed by FlowJo 7.6 software (Tree Star, Inc., Ashland, OR, USA).

Western blot analysis. Proteins from cells were extracted with lysis buffer (Beyotime, Beijing, China) with a $1 \%$ volume of phosphatase inhibitor (Roche Diagnostics, Basel, Switzerland) mixture. Total protein was measured using a standard bicinchoninic acid assay (Pierce Biotechnology, Inc., Rockford, IL, USA). Protein samples were fractioned in a $10 \%$ SDS-polyacrylamide gel and transferred to a polyvinylidene fluoride membrane. After blocking by $5 \%$ skimmed milk for $1 \mathrm{~h}$, western blot analysis was performed with each of the indicated primary antibodies at $4^{\circ} \mathrm{C}$ overnight and secondary antibodies for $1 \mathrm{~h}$. Immunodetection was performed using enhanced electrochemiluminescence.

Statistical analyses. All the data are presented as the means \pm standard deviation. Difference among groups was compared using one-way analysis of variance followed by the least significant difference test. Values of $\mathrm{P}<0.05$ were considered to indicate a statistically significant difference. Experiments of all the groups were performed in triplicate.

\section{Results}

Characterization of BM-derived EPCs and detection of transfected EPCs. The isolated EPCs exhibited a spindle appearance with distinct colony formations by $72 \mathrm{~h}$ (Fig. 1A). The 7-day cultured EPCs were identified by Dil-ac-LDL and FITC-lectin double staining (Fig. 1B). To further confirm the endothelial profile of EPCs, the expression of endothelial markers was examined by immunofluorescent staining. The results showed that the majority of cultured EPCs expressed endothelial markers, including vWF (Fig. 1C) and VEGFR-2 (Fig. 1D), indicating their potential in differentiating into endothelial lineage. In order to enhance the therapeutic potential of EPCs, LV-IL-10-GFP and LV-NC-GFP were transfected into EPCs. GFP-labeled EPCs were detected by a fluorescence microscope, indicating high transfection efficiency (Fig. 1E and F). All the above results confirmed the successful isolation and transfection of BM-derived EPCs. 

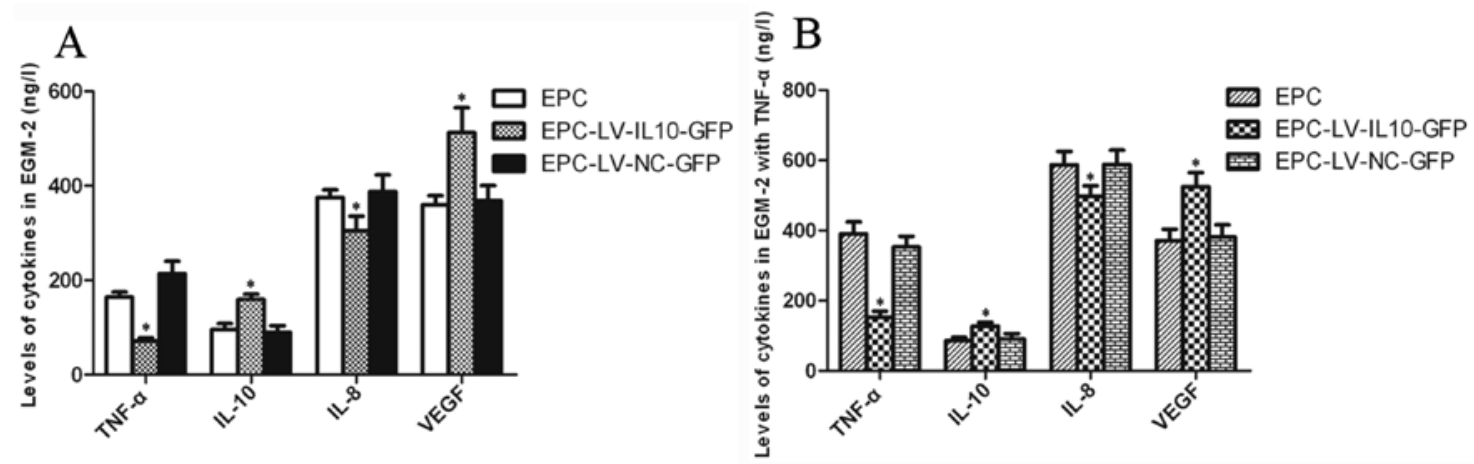

Figure 2. Bar graphs of the ELISA analysis of EPCs. (A) The levels of TNF- $\alpha$ and IL-8 significantly decreased in the EPC-LV-IL-10-GFP group compared to the EPC and EPC-LV-NC-GFP groups, and there was no significant difference between the EPC and EPC-LV-NC-GFP group. By contrast, the levels of IL-10 and VEGF were significantly increased in the EPC-LV-IL-10-GFP group compared to the EPC and EPC-LV-NC-GFP groups, and there was no statistically significant difference between the EPC and EPC-LV-NC-GFP group. (B) ELISA analysis of EGM-2 with TNF- $\alpha$ was consistent with EGM-2 without TNF- $\alpha$ $($ ( $\mathrm{P}<0.05)$. EPCs, endothelial progenitor cells; TNF- $\alpha$, tumor necrosis factor- $\alpha$; IL-8, interleukin-8; LV, lentivirus vector; GFP, green fluorescent protein; NC, noncontain; VEGF, vascular endothelial growth factor; EGM-2, endothelial cell growth medium-2.
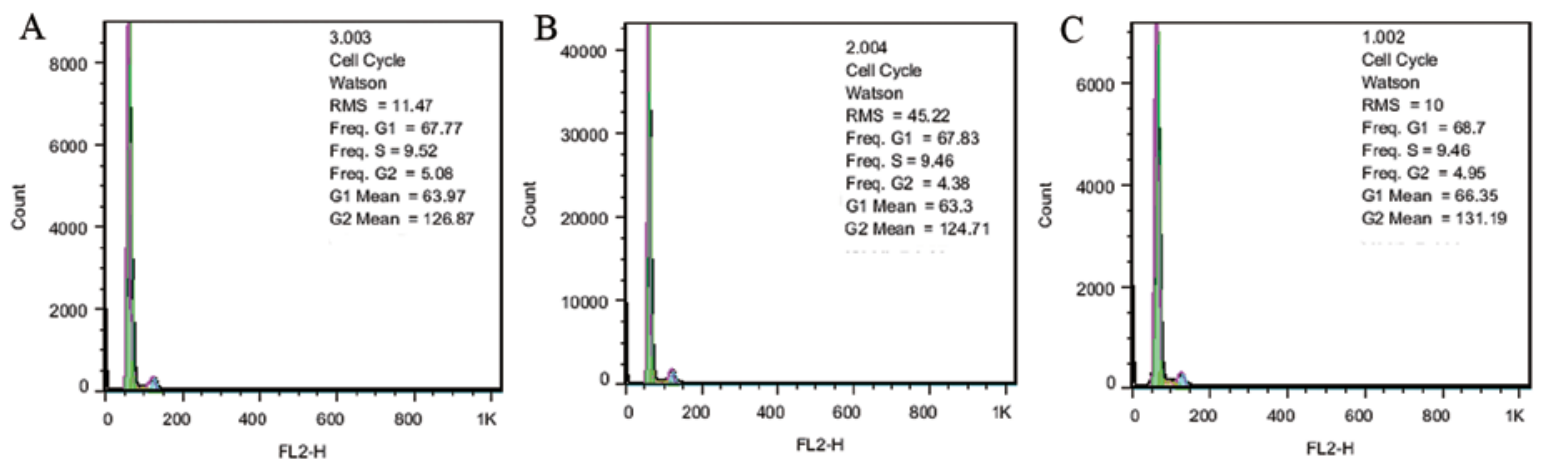

Figure 3. Cell cycle distribution in the EPCs. Cell number histogram plot showing the distribution of cells on the basis of DNA content. (A) EPC, (B) EPC-LV-NC-GFP and (C) EPC-LV-IL-10-GFP groups. EPCs, endothelial progenitor cells; LV, lentivirus vector; NC, noncontain; GFP, green fluorescent protein; IL-10, interleukin-10.

Concentrations of cytokines in EGM-2. The levels of TNF- $\alpha$ and IL-8 were significantly decreased in the EPC-LV-IL-10-GFP group compared to the EPC and EPC-LV-NC-GFP groups $(\mathrm{P}<0.05$ for all), and there was no statistically significant difference between the EPC and EPC-LV-NC-GFP group ( $\mathrm{P}>0.05)$. By contrast, the levels of IL-10 and VEGF were contrasting in association to these. The levels significantly increased in the EPC-LV-IL-10-GFP group compared to the EPC and EPC-LV-NC-GFP groups $(\mathrm{P}<0.05$ for all), and there was no statistically significant difference between the EPC and EPC-LV-NC-GFP groups $(\mathrm{P}>0.05)$ ELISA analysis of EGM-2 with TNF- $\alpha$ were consistent with EGM-2 without TNF- $\alpha$. The bar graphs of the ELISA analysis are shown in Fig. 2. The results showed that IL-10-transfected EPCs were more resistant to an inflammatory stimulus, such as TNF- $\alpha$, leading to the decline of pro-inflammatory factors. Therefore, the function of IL-10-modified EPCs may be improved in the inflammatory environment.

Overexpression of IL-10 modulates EPC function. To explore the role of IL-10 in EPC function, EPCs were transfected with LV-IL-10-GFP and LV-NC-GFP was the control group. Analysis of the cell cycle by flow cytometry with PI/RNase staining indicated that cells in the $\mathrm{S}$ phase were $9.52,9.46$ and $9.46 \%$ in the EPC, EPC-LV-IL-10-GFP and EPC-LV-NC-GFP groups, respectively. The result revealed that there was no significant difference in cell growth between the three groups (Fig. 3). The data showed that the average number of EPCs undergoing migration, adhesion and total lengths of EPCs were similar among the EPC, EPC-LV-IL-10-GFP and EPC-LV-NC-GFP groups without TNF- $\alpha$ (P>0.05 for all) (Figs. 4E, 5 and $6 \mathrm{E}$ ). Further study showed that EPC-LV-IL-10-GFP with TNF- $\alpha$ significantly enhanced EPC migration (Fig. 4), adhesion (Fig. 5) and promoted EPC tube formation (Fig. 6) compared to EPC-LV-NC-GFP with TNF- $\alpha$ and EPC with TNF- $\alpha(\mathrm{P}<0.05$ for all). The bar graph regarding the groups with or without TNF- $\alpha$ are shown in Figs. 4E, 5E and 6E. In summary, these data confirmed that the overexpression of IL-10 had no effect on migration, adhesion, tubule formation and cell growth of EPCs without TNF- $\alpha$. Furthermore, the overexpression of IL-10 improved the function, including the migration, adhesion and tubule formation of EPCs stimulated with TNF- $\alpha$.

Expression of proteins in EPCs. To study the mechanism of IL-10 in EPCs, the expression of MMP-9, VEGF, STAT-3 and p-STAT3 was examined. Western blot analysis revealed that the expression of VEGF, MMP-9 and p-STAT3 significantly increased in the EPC-LV-IL-10-GFP group compared to the other groups. By contrast, STAT-3 expression decreased in 


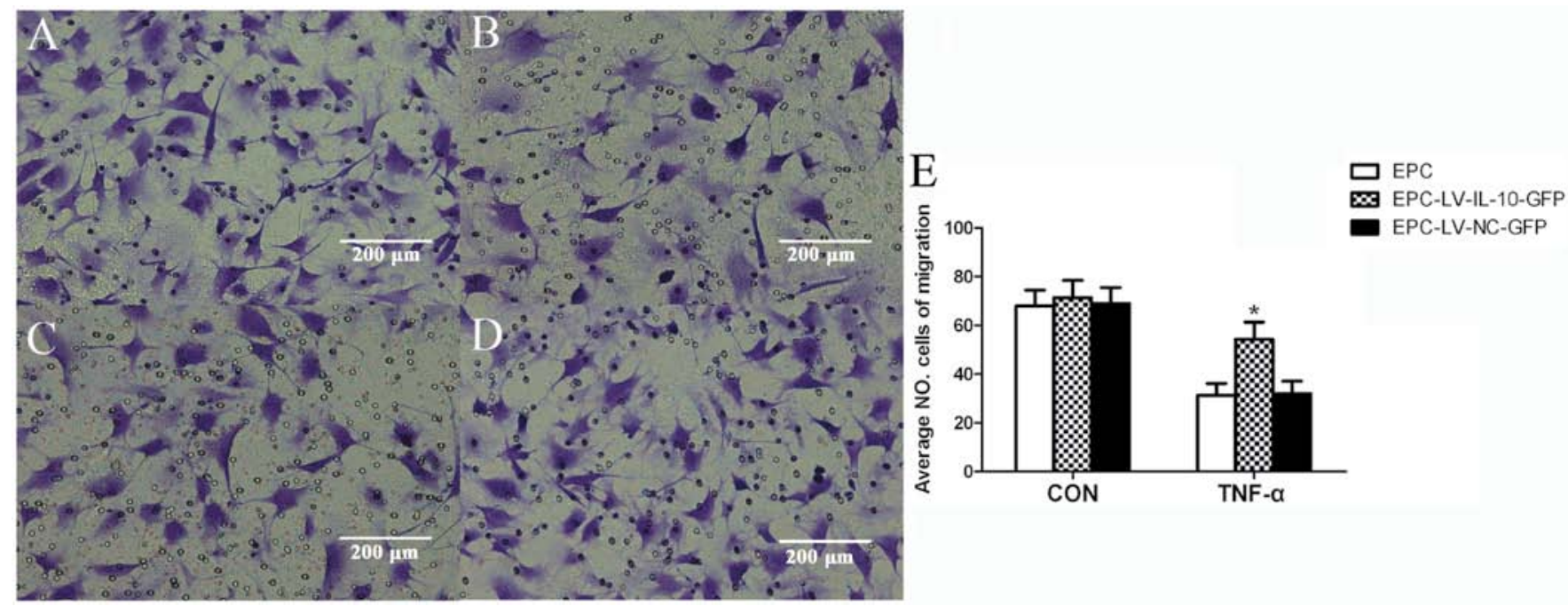

Figure 4. EPC migration (magnification, $\mathrm{x} 200$ ). (A-D) The images show the cells stained with crystal violet on a microporous membrane. The groups of CON are cells in EGM-2 without TNF- $\alpha$; and the groups of TNF- $\alpha$ are cells in EGM-2 with TNF- $\alpha$. (A) EPC without TNF- $\alpha$; (B) EPC with TNF- $\alpha$; (C) EPC-LV-NC-GFP with TNF- $\alpha$; and (D) EPC-LV-IL-10-GFP with TNF- $\alpha$. (E) Bar graph regarding the groups with or without TNF- $\alpha$. The result showed that the average number of EPC undergoing migration was similar to the groups without TNF- $\alpha$. However, EPC-LV-IL-10-GFP with TNF- $\alpha$ significantly enhanced EPC migration compared to EPC-LV-NC-GFP with TNF- $\alpha$ and EPC with TNF- $\alpha$ ("P<0.05 vs. EPC-LV-NC-GFP with TNF- $\alpha$ and EPC with TNF- $\alpha$ ). EPCs, endothelial progenitor cells; EGM-2, endothelial cell growth medium-2; TNF- $\alpha$, tumor necrosis factor- $\alpha$; LV, lentivirus vector; NC, noncontain; GFP, green fluorescent protein; IL-10, interleukin-10.

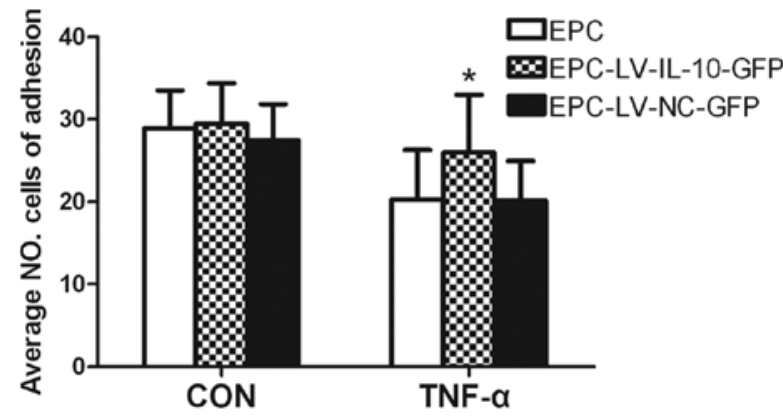

Figure 5. EPC adhesion. The CON group are cells in EGM-2 without TNF- $\alpha$ and the groups of TNF- $\alpha$ are cells in EGM- 2 with TNF- $\alpha$. The result showed that the average number of EPCs undergoing adhesion was similar to groups without TNF- $\alpha$. However, EPC-LV-IL-10-GFP with TNF- $\alpha$ significantly enhanced EPCs adhesion compared to EPC-LV-NC-GFP with TNF- $\alpha$ and EPC with TNF- $\alpha($ P $<0.05)$. EPCs, endothelial progenitor cells; EGM-2, endothelial cell growth medium-2; TNF- $\alpha$, tumor necrosis factor- $\alpha$; LV, lentivirus vector; $\mathrm{NC}$, noncontain; GFP, green fluorescent protein; IL-10, interleukin-10.

the EPC-LV-IL-10-GFP group compared to the other groups (Fig. 7). The results suggested that improvement in the function of IL-10-modified EPCs was possibly through the activation of the STAT3 signaling pathway.

\section{Discussion}

A progenitor cell is a biological cell that, similar to stem cells (SCs), has a tendency to differentiate into a specific type of cell. However, it is already more specific than an SC and is pushed to differentiate into its 'target' cell. EPCs are believed to be derived from the BM and are able to differentiate into mature endothelial cells (ECs). EPCs play a crucial role in the neovascularization of ischemic tissue and in the maintenance of endothelial cell integrity. Since Asahara et al (15) first identified circulating EPCs in 1997, increasing evidence suggests that BM-derived EPCs functionally contribute to neovascularization in several models of tissue injury and remodeling, including wound healing, myocardial ischemia, retinopathy, stroke and peripheral vascular disease. Thus far, techniques aimed at enhancing ex vivo expansion and the therapeutic potential of EPCs, such as epigenetic and genetic modifications of EPCs, are also being extensively studied (16). However, the effect and mechanism remain unclear.

In the past decades, the potential pathogenesis of inflammation in cardiovascular disease (17), lung cancer (18), pulmonary hypertension (19), vascular injury (20) and diabetes (21) has been extensively investigated. Thus, anti-inflammation mediators play a critical role in treatment for diseases. IL-10 is a $35-\mathrm{kDa}$ homodimeric cytokine that is produced by a variety of cell types. IL-10, a potent anti-inflammatory cytokine, attenuates inflammatory response and suppresses various pro-inflammatory mediators (6). Thus, the aim of the present study was to observe the influence of IL-10 overexpression on EPCs and to clarify the possible mechanism. IL-10-modified EPCs may be a potentially therapeutic method for numerous vascular diseases.

In the present study, the data indicate that IL-10 does not influence migration, adhesion, tubule formation and cell growth of EPCs. TNF- $\alpha$ is one of the critical pro-inflammatory cytokines. Peplow (22) reported that TNF- $\alpha$ has an unfavorable influence on cell migration and apoptosis. In order to further study the influence of IL-10 on EPCs, EPC functions were tested under the inflammatory microenvironment induced by TNF- $\alpha$. The results showed that IL-10 enhanced the function of EPCs stimulated with TNF- $\alpha$, including migration, adhesion and tubule formation in vitro. In addition, a previous study demonstrated that EPC was able to carry and express hNIS in glioma following intravenous administration (23). Based on this, it is possible to use EPCs as carrier delivery vehicles or therapeutic genes, which can be administered either systemically or locally. In addition, IL-10-modified EPCs possibly 

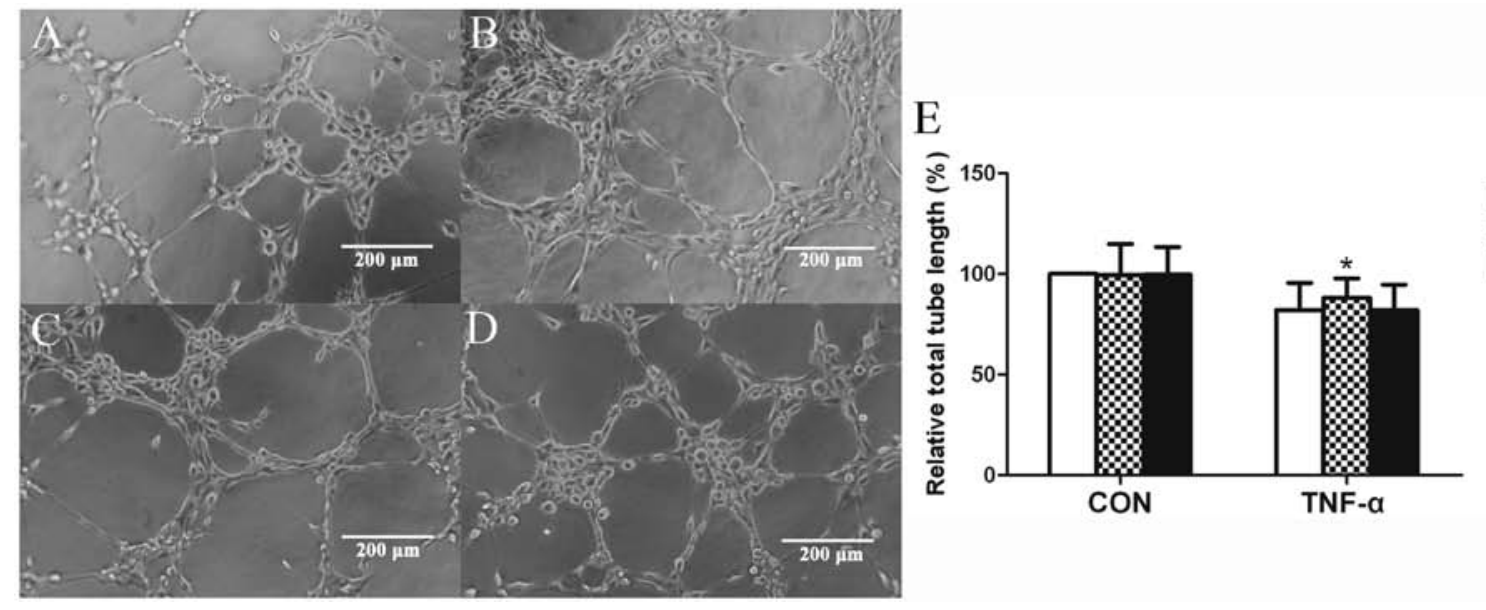

Figure 6. EPC tubulization (magnification, x200). EPCs were seeded onto matrigel to form tubule structures. (A-D) The images demonstrate that cells formed tubule networks. The groups of CON are cells in EGM-2 without TNF- $\alpha$; and the groups of TNF- $\alpha$ are cells in EGM-2 with TNF- $\alpha$. (A) EPC without TNF- $\alpha$; (B) EPC with TNF- $\alpha$; (C) EPC-LV-NC-GFP with TNF- $\alpha$; and (D) EPC-LV-IL-10-GFP with TNF- $\alpha$. (E) Bar graph regarding the groups with or without TNF- $\alpha$. The result showed that the total lengths of EPC tube formation were similar to the groups without TNF- $\alpha$. However, EPC-LV-IL-10-GFP with TNF- $\alpha$ significantly enhanced EPC tubulization compared to EPC-LV-NC-GFP with TNF- $\alpha$ and EPC with TNF- $\alpha$. The total tubular lengths are depicted as percentage change vs. EPC group ("P<0.05 vs. EPC-LV-NC-GFP with TNF- $\alpha$ and EPC with TNF- $\alpha$ ). EPCs, endothelial progenitor cells; EGM-2, endothelial cell growth medium-2; TNF- $\alpha$, tumor necrosis factor- $\alpha$; LV, lentivirus vector; NC, noncontain; GFP, green fluorescent protein; IL-10, interleukin-10.

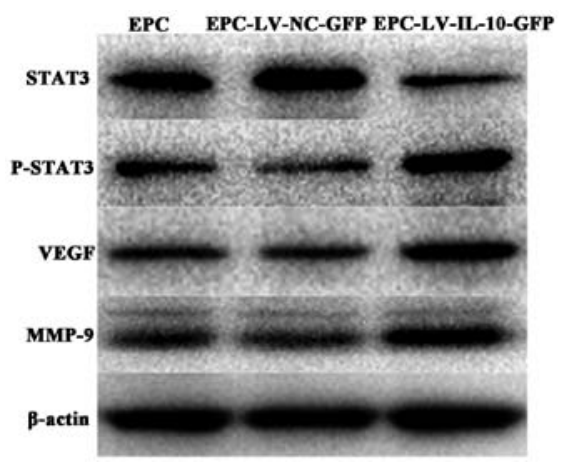

Figure 7. Western blot analysis for the EPCs. The results revealed that the expression of VEGF, MMP-9 and p-STAT3 significantly increased in the EPC-LV-IL-10-GFP group compared to the EPC-LV-NC-GFP and EPC groups. By contrast, STAT-3 expression decreased in the EPC-LV-IL-10-GFP group compared to the EPC-LV-NC-GFP and EPC groups. EPCs, endothelial progenitor cells; VEGF, vascular endothelial growth factor; MMP-9, matrix metalloproteinase 9; p-STAT3, phosphorylated-signal transducer and activator of transcription 3; LV, lentivirus vector; IL-10, interleukin-10; GFP, green fluorescent protein; NC, noncontain.

have a clear positive clinical effect, due to the improvement of the local inflammatory microenvironment and promotion of EPC function.

The IL-10 signaling pathway has been primarily elucidated in monocytes. However, the mechanism of EPCs affected by IL-10 remains unclear. There are numerous studies that exhibit the association between IL-10 and STAT3 $(24,25)$. STAT3 is known to be involved in the development and progression of a number of different types of tumor. The STAT signaling pathway is activated by a diverse array of cytokines and growth factors, and has been indicated in a variety of cellular functions, including inflammatory processes (26). IL-10 dimerizes to bind to a tetramer receptor complex that comprises two molecules of IL-10 receptor 1 (R1) and two molecules of IL-10R2, which permits phosphorylation and dimerization of STAT3. Phosphorylated-STAT3 translocates to the nucleus to activate the downstream target genes. Therefore, we hypothesize that IL-10 promotes the function of EPCs through activating the STAT3 signaling pathway.

Western blot analysis suggests that the expression of VEGF, MMP-9 and p-STAT3 significantly increased in the EPC-LV-IL-10-GFP group compared to the EPC and EPC-LV-NC-GFP groups, whereas the expression of STAT3 is contrasting in association to these. VEGF is the noteworthy candidate for stimulating new vessel formation and vascular hyperpermeability and appears to have important roles for mobilization of EPCs. VEGF stimulates proliferation, migration and survival of endothelial cells, which in turn facilitated the endothelialization and recovery of endothelial function of EPCs (27).

MMPs are well known as important modulators of innate inflammation and are associated with angiogenesis and vascular remodeling. Active MMPs degrade vascular basement membrane and other extracellular matrix (ECM) proteins leading to detaching of ECs, which is necessary for proliferation (28), migration (29) and even apoptosis of ECs (30) and is an initial step of angiogenesis or vascular formation. In addition, MMPs can modify non-ECM molecules, such as VEGFR, to affect EC vitality and behavior (31). In addition, deficiency of MMP-9 attenuates ischemia-induced neovascularization through an impairment of bone marrow-derived EPC adhesion, migration and proangiogenic functions (32). Therefore, the present result suggests that IL-10 promotes the EPC migration, adhesion and tube formation by increasing MMP-9 and VEGF expression, and activating the STAT3 signaling pathway.

In conclusion, the present study demonstrated that the overexpression of IL-10 had no effect on migration, adhesion, tubule formation and cell growth of EPCs without stimulation with TNF- $\alpha$. Furthermore, in the EPCs stimulated with TNF- $\alpha$, the overexpression of IL-10 improved EPC function, including migration, adhesion and tubule formation through activating the STAT3 signaling pathway. The delivery of IL-10-modified EPCs to the sites of the different lesions may be a novel therapeutic target. 


\section{Acknowledgements}

The present study was supported by the National Natural Science Foundation of China (grant nos. 81371038 and 91442124).

\section{References}

1. Huang H, Huang F and Huang JP: Transplantation of bone marrow-derived endothelial progenitor cells overexpressing Delta-like-4 enhances functional neovascularization in ischemic myocardium. Mol Med Rep 8: 1556-1562, 2013.

2. Barsotti MC, Santoni T, Picoi ME, et al: Endothelial progenitor cell homing in human myocardium in patients with coronary artery disease. Int J Cardiol 15: 516-517, 2014.

3. Long J, Wang S, Zhang Y, Liu X, Zhang H and Wang S: The therapeutic effect of vascular endothelial growth factor gene - or heme oxygenase-1 gene-modified endothelialprogenitor cells on neovascularization of rat hindlimb ischemia model. J Vasc Surg 58: 756-765, 2013.

4. Roncalli JG, Tongers J, Renault MA and Losordo DW: Endothelial progenitor cells in regenerative medicine and cancer: a decade of research. Trends Biotechnol 26: 276-283, 2008.

5. Grisar J, Aletaha D, Steiner CW, et al: Depletion of endothelial progenitor cells in the peripheral blood of patients with rheumatoid arthritis. Circulation 111: 204-211, 2005.

6. Krishnamurthy P, Lambers E, Verma S, Thorne T, Qin G Losordo DW and Kishore R: Myocardial knockdown of mRNA-stabilizing protein $\mathrm{HuR}$ attenuates post-mi inflammatory response and left ventricular dysfunction in IL-10-null mice. FASEB J 24: 2484-2494, 2010.

7. Krishnamurthy P, Rajasingh J, Lambers E, Qin G, Losordo DW and Kishore R: Il-10 inhibits inflammation and attenuates left ventricular remodeling after myocardial infarction via activation of STAT3 and suppression of HuR. Circ Res 104: e9-e18, 2009.

8. Yao L, Huang K, Huang D, Wang J, Guo H and Liao Y. Acute myocardial infarction induced increases in plasma tumor necrosis factor-alpha and interleukin-10 are associated with the activation of poly (ADP-ribose) polymerase of circulating mononuclear cell. Int J Cardiol 123: 366-368, 2008.

9. Jui HY, Lin CH, Hsu WT, et al: Autologous mesenchymal stem cells prevent transplant arteriosclerosis by enhancing local expression of interleukin-10, interferon-gamma, and indoleamine 2,3-dioxygenase. Cell Transplant 21: 971-984, 2012.

10. Kawamoto A, Iwasaki H, Kusano K, et al: CD34-positive cells exhibit increased potency and safety for therapeutic neovascularization after myocardial infarction compared with total mononuclear cells. Circulation 114: 2163-2169, 2006.

11. Zhao W, Li JJ, Cao DY, et al: Intravenous injection of mesenchymal stem cells is effective in treating liver fibrosis. World J Gastroenterol 18: 1048-1058, 2012.

12. Krishnamurthy $\mathrm{P}$, Thal M, Verma S, et al: Interleukin-10 deficiency impairs bone marrow-derived endothelial progenitor cell survival and function in ischemic myocardium. Circ Res 109: 1280-1289, 2011.

13. Chen DD, Dong YG, Yuan H and Chen AF: Endothelin 1 activation of endothelin A receptor/NADPH oxidase pathway and diminished antioxidants critically contribute to endothelial progenitor cell reduction and dysfunction in salt-sensitive hypertension. Hypertension 59: 1037-1043, 2012.

14. Marrotte EJ, Chen DD, Hakim JS and Chen AF: Manganese superoxide dismutase expression in endothelial progenitor cells accelerates wound healing in diabetic mice. J Clin Invest 120 : 4207-4219, 2010.
15. Asahara T, Murohara T, Sullivan A, et al: Isolation of putative progenitor endothelial cells for angiogenesis. Science 275 : 964-967, 1997.

16. PengLH,Tsang SY,Tabata Y and GaoJQ: Genetically-manipulated adult stem cells as therapeutic agents and gene delivery vehicle for wound repair and regeneration. J Control Release 157: 321-330, 2012.

17. Golia E, Limongelli G, Natale F, et al: Inflammation and cardiovascular disease: from pathogenesis to therapeutic target. Curr Atheroscler Rep 16: 435, 2014.

18. $\mathrm{Pu} \mathrm{X}$, Wang L, Chang JY, et al: Inflammation-related genetic variants predict toxicity following definitive radiotherapy for lung cancer. Clin Pharmacol Ther Jul 23, 2014 (Epub ahead of print).

19. Guignabert $C$ and Dorfmuller P: Pathology and pathobiology of pulmonary hypertension. Semin Respir Crit Care Med 34: 551-559, 2013.

20. Inoue T, Croce K, Morooka T, Sakuma M, Node K and Simon DI: Vascular inflammation and repair: implications for re-endothelialization, restenosis, and stent thrombosis. JACC Cardiovasc Interv 4: 1057-1066, 2011.

21. Tang $\mathbf{J}$ and Kern TS: Inflammation in diabetic retinopathy. Prog Retin Eye Res 30: 343-358, 2011.

22. Peplow PV: Influence of growth factors and cytokines on angiogenic function of endothelial progenitor cells: a review of in vitro human studies. Growth Factors 32: 83-116, 2014.

23. Palenski TL, Sorenson CM and Sheibani N: Inflammatory cytokine-specific alterations in retinal endothelial cell function. Microvasc Res 89: 57-69, 2013.

24. Lafarge S, Hamzeh-Cognasse H, Chavarin P, Genin C, Garraud $\mathrm{O}$ and Cognasse F: A flow cytometry technique to study intracellular signals NF-kappaB and STAT3 in peripheral blood mononuclear cells. BMC Mol Biol 8: 64, 2007.

25. Maher K, Završnik J, Jerič-Kokelj B, Vasiljeva O, Turk B and Kopitar-Jerala N: Decreased IL-10 expression in stefin B-deficient macrophages is regulated by the MAP kinase and STAT-3 signaling pathways. FEBS Lett 588: 720-726, 2014.

26. Leonard WJ and O'Shea JJ: Jaks and STATS: biological implications. Annu. Rev Immunol 16: 293-322, 1998.

27. Jin K, Kashiwagi K, Iizuka Y, Tanaka Y, Imai M and Tsukahara S: Matrix metalloproteinases in human diabetic and nondiabetic vitreous. Retina 21: 28-33, 2001.

28. Mazor R, Alsaigh T, Shaked H, et al: Matrix metalloproteinase-1-mediated up-regulation of vascular endothelial growth factor-2 in endothelial cells. J Biol Chem 288: 598-607, 2013.

29. Genís L, Gonzalo P, Tutor AS, et al: Functional interplay between endothelial nitric oxide synthase and membrane type 1-matrix metalloproteinase in migrating endothelial cells. Blood 110: 2916-2923, 2007

30. Shapiro S, Khodalev O, Bitterman H and Auslender R and Lahat N: Different activation forms of MMP-2 oppositely affect the fate of endothelial cells. Am J Physiol Cell Physiol 298: C942-C951, 2010.

31. Ito TK, Ishii G, Saito S, Yano K, Hoshino A, Suzuki T and Ochiai A: Degradation of soluble vegf receptor-1 by MMP-7 allows VEGF access to endothelial cells. Blood 113: 2363-2369, 2009.

32. Huang PH, Chen YH, Wang $\mathrm{CH}$, et al: Matrix metalloproteinase- 9 is essential for ischemia-induced neovascularization by modulating bone marrow-derived endothelial progenitor cells. Arterioscler Thromb Vasc Biol 29: 1179-1184, 2009. 